\title{
Altered Relationship between Working Memory and Brain Microstructure after Mild Traumatic Brain Injury
}

\author{
(D) S. Chung, (D)X. Wang, (D)E. Fieremans, (D).F. Rath, (D)P. Amorapanth, (D)F.-Y.A. Foo, (D) C.J. Morton, DD.S. Novikov, \\ (D) S.R. Flanagan, and DY.W. Lui
}

\begin{abstract}
BACKGROUND AND PURPOSE: Working memory impairment is one of the most troubling and persistent symptoms after mild traumatic brain injury (MTBI). Here we investigate how working memory deficits relate to detectable WM microstructural injuries to discover robust biomarkers that allow early identification of patients with MTBI at the highest risk of working memory impairment.
\end{abstract}

MATERIALS AND METHODS: Multi-shell diffusion MR imaging was performed on a 3T scanner with 5 b-values. Diffusion metrics of fractional anisotropy, diffusivity and kurtosis (mean, radial, axial), and WM tract integrity were calculated. Auditory-verbal working memory was assessed using the Wechsler Adult Intelligence Scale, 4th ed, subtests: 1) Digit Span including Forward, Backward, and Sequencing; and 2) Letter-Number Sequencing. We studied 19 patients with MTBI within 4 weeks of injury and 20 healthy controls. Tract-Based Spatial Statistics and ROI analyses were performed to reveal possible correlations between diffusion metrics and working memory performance, with age and sex as covariates.

RESULTS: ROI analysis found a significant positive correlation between axial kurtosis and Digit Span Backward in MTBI (Pearson $r=0.69$, corrected $P=.04$ ), mainly present in the right superior longitudinal fasciculus, which was not observed in healthy controls. Patients with MTBI also appeared to lose the normal associations typically seen in fractional anisotropy and axonal water fraction with Letter-Number Sequencing. Tract-Based Spatial Statistics results also support our findings.

CONCLUSIONS: Differences between patients with MTBI and healthy controls with regard to the relationship between microstructure measures and working memory performance may relate to known axonal perturbations occurring after injury.

ABBREVIATIONS: $A K=$ axial kurtosis; $A W F=$ axonal water fraction; bCC = body of the corpus callosum; DKI = diffusional kurtosis imaging; DSF = Digit Span Forward; DSB = Digit Span Backward; DSS = Digit Span Sequencing; FA = fractional anisotropy; gCC = genu of the corpus callosum; HC = healthy control; LNS = Letter-Number Sequencing; MTBI = mild traumatic brain injury; $\mathrm{PCR}=$ posterior corona radiata; $\mathrm{SCC}=$ splenium of the corpus callosum; $\mathrm{SCR}=$ superior corona radiata; $\mathrm{SLF}=$ superior longitudinal fasciculus; WMTI = white matter tract integrity; WAIS = Wechsler Adult Intelligence Scale; WRAT = Wide Range Achievement Test

M ild traumatic brain injury (MTBI) is an important public health problem with many serious consequences. ${ }^{1,2}$ While most patients with MTBI recover symptomatically within 2-3 weeks after injury, at least $15 \%$ of patients report persistent cognitive symptoms that are an important source of distress and disability after injury. ${ }^{3-5}$ Now an important body of work reveals MTBI-related WM injury using DTI $^{6-8}$ and diffusional kurtosis

Received May 15, 2019; accepted after revision June 19.

From the Center for Advanced Imaging Innovation and Research \& Bernard and Irene Schwartz Center for Biomedical Imaging, Department of Radiology (S.C.,

X.W., E.F., C.J.M., D.S.N., Y.W.L.), and Department of Rehabilitation Medicine (J.F.R., P.A., S.R.F.), New York University School of Medicine, New York, New York; and Department of Neurology (F.-Y.A.F.), New York University Langone Health, New York, New York.

This work was supported, in part, by grant funding from the National Institutes of Health/National Institute for Neurological Disorders and Stroke: R01 NS039135-11 and R21 NS090349. This work was also performed under the rubric of the Center for Advanced Imaging Innovation and Research (www.cai2r.net), a National Institute of Biomedical Imaging and Bioengineering, Biomedical Technology Resource Center (NIH P41 EB017183). imaging (DKI). ${ }^{9,10}$ More recently, WM tract integrity (WMTI) metrics derived from an advanced compartmental diffusion WM mode ${ }^{11}$ have been proposed to describe microstructural characteristics in the intra- and extra-axonal environments of WM, including axonal water fraction (AWF), intra-axonal diffusivity, and extra-axonal axial and radial diffusivities.

One of major barriers to applying such findings to clinical cohorts is that the disorder is extremely heterogeneous and most current studies group clinically heterogeneous cohorts of patients with MTBI together, representing a broad spectrum of clinical symptoms. Thus, there is a specific need to understand domainspecific symptoms as they relate to detectable microstructural injuries, to better understand patient-specific injury and recovery.

\footnotetext{
Please address correspondence to Sohae Chung, PhD, 660 1st Ave, 4th floor, New York, NY 10016; e-mail: sohae.chung@nyulangone.org

- - Indicates open access to non-subscribers at www.ajnr.org

http://dx.doi.org/10.3174/ajnr.A6146
} 
One of the most common and clinically significant symptoms in patients with MTBI is deficits in working memory, ${ }^{3,4,12-15}$ which often negatively affect quality of life. ${ }^{16}$ This result comes as no surprise because working memory, which involves the capacity to temporarily store and manipulate information in pursuit of a goal, is at the core of critical cognitive functions such as comprehension, learning, reasoning, and decision-making. ${ }^{17}$ Working memory is conceptualized as comprising 3 main components: the central executive, responsible for manipulation of information and allocation of attention and processing resources, and 2 maintenance systems, the phonologic loop (verbal and auditory information) and the visuospatial sketchpad (visual and spatial information). ${ }^{18-20}$ There have been a few studies showing associations of working memory performance with measures of WM microstructure such as fractional anisotropy $(\mathrm{FA})^{21}$ and $\mathrm{AWF}^{22-24}$ in healthy individuals, believed to relate to differences in axon volume and myelination. However, such associations have not yet been investigated in patients with MTBI.

Here, we hypothesize that WM injury in patients with MTBI can affect the relationship between microstructural changes to the WM and working memory performance. To test this hypothesis, we investigated the relationship between WM microstructural changes assessed using diffusion MR imaging (DTI, DKI, WMTI) and a set of Wechsler Adult Intelligence Scale, 4th ed (WAIS-IV) ${ }^{25}$ subtests tapping auditory-verbal working memory functions in patients with MTBI, comparing them against healthy controls (HCs). We also performed subgroup analyses based on working memory performance and time since injury.

\section{MATERIALS AND METHODS Study Population}

This study was approved by our institutional review board at New York University Langone Health. All experiments were performed in accordance with relevant guidelines and regulations, and written informed consent was provided by all subjects before the procedure. We prospectively recruited subjects who were seen for clinical care in the Emergency Department or Institutional Concussion Center. Inclusion criteria were the following: 1) adult individuals in the age range of 18-65 years; 2) diagnostic MTBI criteria defined by the American Congress of Rehabilitation Medicine, ${ }^{26}$ including either loss of consciousness of $<30$ minutes or altered consciousness at time of the accident and a Glasgow Outcome Score of 13-15; and 3) injury within 4 weeks. We excluded patients with the following: 1) a history of traumatic brain injury, neurologic illness, or psychiatric disorder; 2) a history of participation in organized contact sports; and 3) any contraindication to MR imaging. We also further excluded non-native English speakers and non-right-handed individuals to avoid any potential confounding effects of language and handedness. We studied 19 patients with MTBI (mean age, $30 \pm 7$ years; age range, $22-45$ years; average time since injury, 16 days; 9 men) and $20 \mathrm{HCs}$ (mean age, $33 \pm 10$ years; age range, $19-65$ years; 9 men). For all subjects, formal neurocognitive tests including the WAIS-IV working memory subtests were performed, and MR images were acquired within 1 day of neurocognitive tests. Additionally, to characterize subjects, the Wide Range Achievement Test, 4th ed, Word Reading subtest (WRAT-4) was performed, and the scores were con- verted to intelligence quotient scores as a brief measure of intelligence. Subgroups of the subjects in this study were previously included in 2 works with nonoverlapping hypotheses. ${ }^{24,27}$

\section{MR Imaging Protocol}

MR imaging was performed using a 3T MR imaging scanner (Magnetom Skyra; Siemens, Erlangen, Germany). Diffusion imaging was performed with 5 b-values $(250,1000,1500,2000$, and $\left.2500 \mathrm{~s} / \mathrm{mm}^{2}\right)$ using 5 diffusion-encoding direction schemes $(6,20$, 20,30 , and 60 , respectively). Three images with $b=0 \mathrm{~s} / \mathrm{mm}^{2}$ were also acquired. Multiband (factor of 2$)^{28}$ EPI was used for accelerated acquisitions with an anterior-posterior phase-encoding direction. Other parameters included the following: $\mathrm{FOV}=220 \times$ $220 \mathrm{~mm}$, acquisition matrix $=88 \times 88$, number of slices $=56$, image resolution $=2.5 \times 2.5 \times 2.5 \mathrm{~mm}^{3}$, TR/TE $=4900 / 95 \mathrm{~ms}$, bandwidth $=2104 \mathrm{~Hz} /$ pixel, a generalized autocalibrating partially parallel acquisition factor of 2 . An additional image with $b=0 \mathrm{~s} / \mathrm{mm}^{2}$ with a reversed phase-encoding direction was acquired for geometric artifact correction. The total scan time was 12 minutes.

\section{Working Memory Assessment}

Working memory was assessed with age-appropriate WAIS-IV subtests, ${ }^{25}$ which included Digit Span and Letter-Number Sequencing (LNS). In the Digit Span Forward (DSF) task, examinees repeat a sequence of numbers read to them. In the Digit Span Backward (DSB), the same procedure is followed, except that examinees repeat the numbers in reverse order. In the Digit Span Sequencing (DSS), examinees repeat the numbers in ascending order. In the LNS task, examinees separate numbers from letters and state in ascending/alphabetical order a mixed sequence of numbers and letters read to them. Raw scores were converted into standardized age-corrected $z$ scores with a zero mean and a unitary variation, ${ }^{25}$ with higher scores indicating better performance.

\section{Image Analyses}

Diffusion Image Processing. The diffusion images underwent the preprocessing steps including Marchenko-Pastur principal component analysis denoising, ${ }^{29}$ Gibbs correction, ${ }^{30}$ distortion correction with the topup command in FSL (https://fsl.fmrib.ox. ac.uk/fsl/fslwiki/topup), eddy current distortion and motion correction with the eddy command in FSL (https://fsl.fmrib.ox. ac.uk/fsl/fslwiki/eddy), and outlier detection. ${ }^{31}$ In total, 11 diffusion metrics including DTI (FA, mean diffusivity [MD], axial diffusivity $[A D]$, radial diffusivity $[R D]$ ), DKI (mean kurtosis $[M K]$, axial kurtosis $[\mathrm{AK}]$, radial kurtosis $[\mathrm{RK}]$ ), and WMTI (AWF, intra-axonal diffusivity, extra-axonal axial diffusivity, and extra-axonal radial diffusivity) metrics were calculated using in-house software developed in Matlab R2017a (MathWorks, Natick, Massachusetts).

Tract-Based Spatial Statistics. We used the standard Tract-Based Spatial Statistics (TBSS; http://fsl.fmrib.ox.ac.uk/fsl/fslwiki/ TBSS $^{32}$ to reveal possible correlations between working memory test $z$ scores and diffusion metrics. Briefly, subject FA maps were normalized to the FA template through a nonlinear coregistration, and voxelwise statistical analysis was performed on FA val- 
ues projected onto the FA skeleton by looking for local maximum values perpendicular to the skeleton using a permutation-based nonparametric testing randomize command in FSL (https:// fsl.fmrib.ox.ac.uk/fsl/fslwiki/Randomise/UserGuide) with the threshold-free cluster enhancement (TFCE) option. All other parametric maps underwent the same transformations and processes. The tract skeleton was thresholded at an FA of 0.2 for DTI and DKI metrics. For WMTI metrics, analysis was restricted to WM regions consisting of single-fiber orientations (FA threshold of 0.4 ), as recommended. ${ }^{11,33}$ Age and sex were included as covariates. The number of permutations was set to 5000 .

ROI Analysis. ROI analysis was performed on 18 major WM tracts, including genu/body/splenium of the corpus callosum ( $\mathrm{gCC} / \mathrm{bCC} / \mathrm{sCC}$ ), right and left anterior/posterior limb of the internal capsule, right and left anterior/superior ( $\mathrm{sCR}) /$ posterior corona radiata (pCR), right and left cingulum, right and left superior longitudinal fasciculus (SLF), and whole WM. ROI regions were generated on the basis of the Johns Hopkins University ICBM-DTI-81 WM labels atlas. ${ }^{34}$ Briefly, all subjects' FA maps were nonlinearly registered to the FA template; then, a "reversed warping" procedure was performed to assign the atlas labels to each subject's space. The ROIs in each subject's space were manually corrected if necessary. For each ROI, the mean value was obtained only in voxels with FA $\geq 0.2$ for DTI and DKI metrics and with $\mathrm{FA} \geq 0.4$ for WMTI metrics, to restrict analysis to WM regions, as recommended. ${ }^{11,33}$

\section{Statistical Analysis}

The MANCOVA was used to test group differences in the length of education and WRAT-4 intelligence quotient scores, with age and sex as covariates, using SPSS Statistics software, Version 25.0 (IBM, Armonk, New York). Results were considered significant for $P<.05$.

For TBSS, a statistical threshold level of $P<.05$ was applied after family-wise error correction for multiple comparisons.

For ROI analysis, both the Pearson partial correlation and Spearman rank correlation were performed to measure the associations between diffusion metrics and WAIS-IV subtest scores in each ROI, using SPSS. Age and sex were included as covariates. All $P$ values were corrected for multiple comparisons using the Benjamini-Hochberg correction. A statistical threshold level of corrected $P<.05$ was used. Correlation coefficients were calculated. We also used Fisher R-to-Z transformation ${ }^{35}$ to test for differences in between-group correlations.

On the basis of the ROI analysis results, specific regions demonstrating significant correlations with working memory performance were further interrogated by dividing subjects into subgroups based on their working memory test $z$ scores $(<1, \geq 1)$ and time since injury ( $<2$ weeks, $2-4$ weeks). Subgroup comparisons were performed using MANCOVA, with age as a covariate. Results were considered significant at $P<.05$.

\section{RESULTS}

The average length of education for patients with MTBI was $15.5 \pm 1.7$ years, not statistically different from that in healthy controls ( $16.3 \pm 1.8$ years). Also, WRAT-4 intelligence quotient scores were not statistically different between MTBI (108.5 \pm

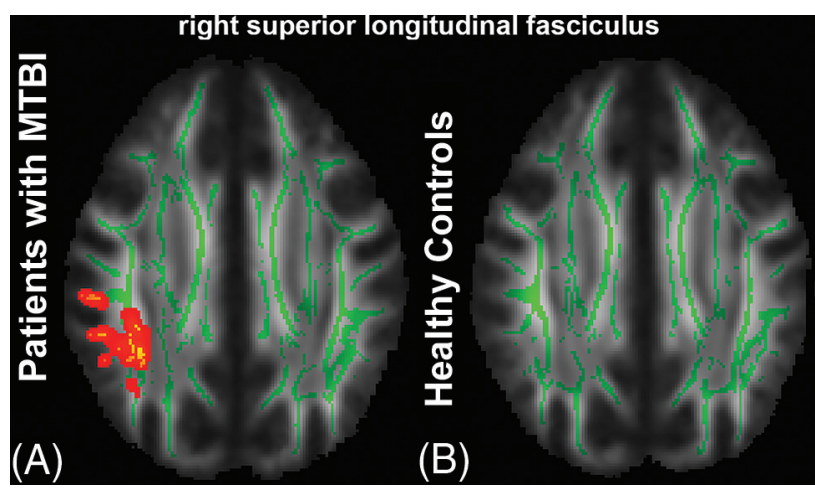

FIG 1. TBSS results show a significant positive correlation between $A K$ and DSB. The mean FA skeleton (green) is overlaid on the mean FA map. Significantly correlated voxels (corrected $P<.05$ ) are shown in the heat map overlay in the right SLF in the MTBI group $(A)$, but are not seen in the $\mathrm{HC}$ group $(B)$.

$12.0)$ and HC (113.6 \pm 14.3$)$ groups. WAIS-IV subtests were not significantly correlated with age and length of education for MTBI and $\mathrm{HC}$ groups, except for positive correlations between the length of education and DSS in HCs $(P=.03)$.

From TBSS analysis, in the MTBI group, we found a significant positive correlation between AK and DSB, primarily in the right SLF (Fig 1A), which was not present in the HC group (Fig $1 B)$. Most interesting, we found complete loss of relationships between FA and LNS in the MTBI group (Fig 2A), while a significant correlation was shown in the HC group (Fig 2B), most notably in parietal WM, sCR/pCR, bCC/sCC, and SLF. We also found no significant correlation between AWF and LNS in the MTBI group (Fig $3 A$ ), while there were multiple areas showing a statistically significant positive correlation between AWF and LNS in the HC group, involving parietal WM, sCR/pCR, and $\mathrm{bCC} / \mathrm{sCC}$ (Fig 3B). No other diffusion metrics showed areas of significant correlation surviving correction for multiple comparisons.

ROI analysis also found a significant correlation between AK and DSB in the right SLF in the MTBI group (Pearson $R=0.69$, $P=0.04$; Spearman $\rho=0.75, P=0.01$ ) that was not present in the $\mathrm{HC}$ group. The correlation coefficients observed in the MTBI and HC groups were significantly different (Fisher R-to-Z transformation, $P=.01$ ). On the other hand, we also observed loss of relationships between FA and LNS in the MTBI group but found a significant positive correlation in the right $\mathrm{pCR}$ in the HC group (Pearson $R=0.67, P=0.04$; Spearman $\rho=0.57, P=0.09$ ). The correlation coefficients observed in the MTBI and HC groups were not significantly different, but there was a trend toward significance (Fisher R-to-Z transformation, $P=.06$ ). No other significant correlations were found after correction for multiple comparisons. These results are summarized in Table 1.

On the basis of the results of the ROI analysis, subjects were further divided into subgroups according to their working memory test $z$ scores $(<1$ or $\geq 1)$ and time since injury ( $<2$ weeks or $2-4$ weeks). Details of the subgroup characteristics are given in Tables 2 and 3. With regard to subgroup analysis, we found a significant difference in AK between the MTBI subgroup of 2-4 weeks from injury with DSB $<1$ and the MTBI subgroup 
of 2-4 weeks from injury with DSB $\geq 1$ within the right SLF (Fig $4 A$ ). Significant differences in FA were shown between the MTBI subgroup of $<2$ weeks of injury with LNS $<1$ and the
HC subgroup with LNS $<1$, and between the HC subgroup with LNS $<1$ and the HC subgroup with LNS $\geq 1$, within the right pCR (Fig 4B).

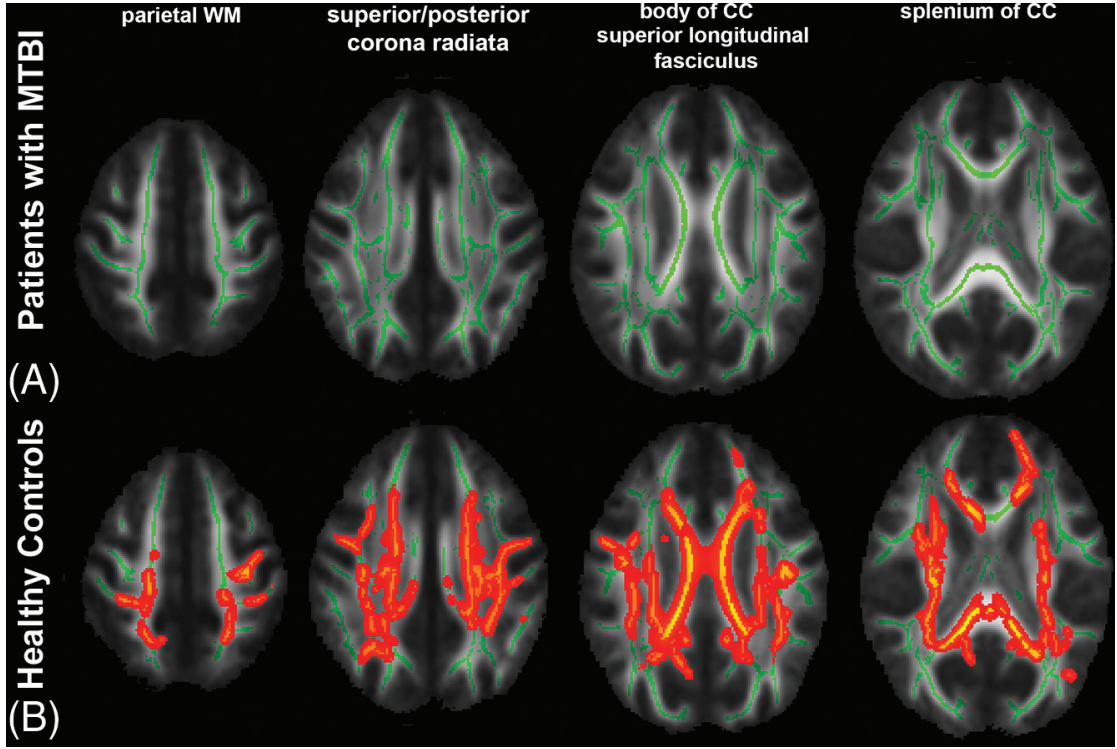

FIG 2. TBSS results show a significant positive correlation between FA and LNS. The mean FA skeleton (green) is overlaid on the mean FA map. Significantly correlated voxels (corrected $P<$ .05 ) are shown in heat map overlay. $A$, In the MTBI group, no correlation was found. $B$, In the HC group, significantly correlated voxels involve the parietal WM, sCR/pCR, bCC/sCC, and SLF.

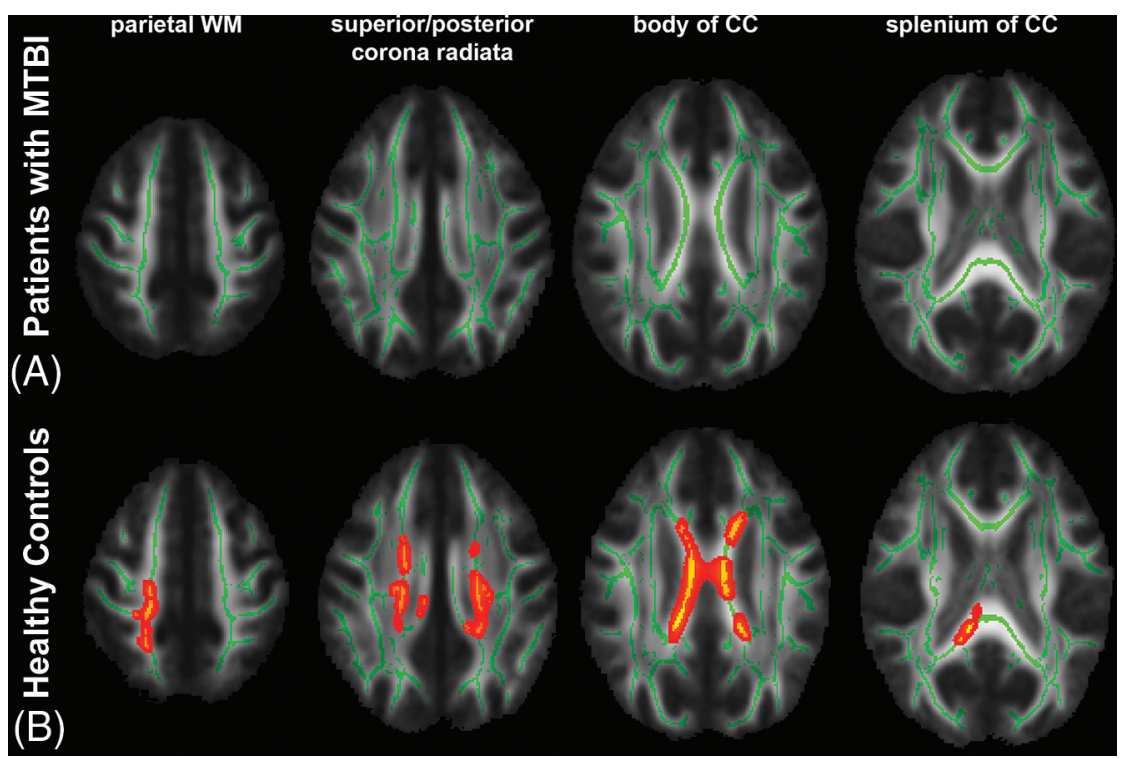

FIG 3. TBSS results show a significant positive correlation between AWF and LNS. The mean FA skeleton (green) is overlaid on the mean FA map. Significantly correlated voxels (corrected $P<$ $.05)$ are shown in the heat map overlay. $A$, In the MTBI group, no correlation was found. $B$, In the $\mathrm{HC}$ group, significantly correlated voxels involve the parietal WM, sCR/pCR, and bCC/sCC.

\section{DISCUSSION}

This study shows significant differences in the relationships between specific WM microstructural markers and auditory-verbal working memory performance between patients with MTBI and healthy controls. Specifically, in the MTBI group, a significant correlation between AK and DSB was present in the right SLF, while the HC group demonstrated no such relationship (Fig 1), a finding observed using both TBSS and ROI analyses. Patients with MTBI also appear to lose the normal associations seen in controls between diffusion metrics (FA, AWF) and LNS (Figs 2 and 3). Furthermore, subgroup analyses also showed a significant difference in AK between the MTBI subgroup of 2-4 weeks of injury with DSB $<1$ and the MTBI subgroup of 2-4 weeks of injury with $\mathrm{DSB} \geq 1$. A significant difference was also found between the MTBI subgroup of $<2$ weeks of injury and the HC subgroup, both having LNS $<1$.

Notably, the relationship between AK and DSB in patients with MTBI is mainly present in the right SLF (Fig 1), a structure critical for attention, memory, emotion, and language, ${ }^{36,37}$ linking frontoparietal WM regions critical to working memory. ${ }^{38-40}$ In particular, the right SLF is critical for attention, ${ }^{41}$ visuospatial function, ${ }^{42}$ and short-term memory. ${ }^{43}$ Previously, similar results were reported showing relations between the SLF and working memory deficits assessed by FA and the visual 2-back d-prime index in patients with severe and diffuse traumatic brain injury. ${ }^{44}$ In this study, we found decreased $\mathrm{AK}$ in the right SLF, reflecting decreased tissue complexity along the long axis of the axon ${ }^{45}$ in patients with MTBI with

Table 1: Results of the ROI analysis showing significant positive correlations between AK and DSB in the MTBI group, and between FA and LNS in the HC group

\begin{tabular}{|c|c|c|c|c|c|c|c|c|c|c|}
\hline \multirow[b]{3}{*}{ Diffusion Metrics vs Working Memory } & \multirow[b]{3}{*}{ ROI } & \multicolumn{4}{|c|}{ MTBI } & \multicolumn{4}{|c|}{$\mathrm{HC}$} & \multirow[b]{3}{*}{ Fisher R-to-Z $(P)$} \\
\hline & & \multicolumn{2}{|c|}{ Pearson } & \multicolumn{2}{|c|}{ Spearman } & \multicolumn{2}{|c|}{ Pearson } & \multicolumn{2}{|c|}{ Spearman } & \\
\hline & & $R$ & $P^{\mathrm{a}}$ & $\rho$ & $P^{a}$ & $R$ & $P^{a}$ & $\rho$ & $P^{\mathrm{a}}$ & \\
\hline AK vs DSB & Right SLF & $0.69^{\mathrm{b}}$ & $.04^{\mathrm{b}}$ & $.75^{\mathrm{b}}$ & $.01^{\mathrm{b}}$ & 0.04 & 1.20 & 0.11 & 1.48 & $2.32^{\mathrm{b}}(.01)^{\mathrm{b}}$ \\
\hline FA vs LNS & Right $\mathrm{pCR}$ & 0.25 & 2.29 & .24 & .8 & $0.67^{b}$ & $.04^{\mathrm{b}}$ & 0.57 & .09 & $-1.59(.06)$ \\
\hline
\end{tabular}

${ }^{a}$ Corrected $P$ values after Benjamini-Hochberg correction for multiple comparison.

b Significant results. 
Table 2: Subgroup characteristics defined by their working memory test $z$ scores (LNS $<1$ or $\geq 1$ ) and time since injury ( $<2$ weeks or $2-4$ weeks)

\begin{tabular}{|c|c|c|c|c|c|c|}
\hline & \multicolumn{3}{|c|}{ LNS $<1$} & \multicolumn{3}{|c|}{ LNS $\geq 1$} \\
\hline & MTBI (<2 Weeks) & MTBI (2-4 Weeks) & $\mathrm{HC}$ & MTBI (<2 Weeks) & MTBI (2-4 Weeks) & $\mathrm{HC}$ \\
\hline No. & 5 & 10 & 9 & 3 & 1 & 11 \\
\hline Age & $31 \pm 6$ & $31 \pm 9$ & $31 \pm 8$ & $25 \pm 3$ & 31 & $35 \pm 12$ \\
\hline LNS & $-0.13 \pm 0.51$ & $0.07 \pm 0.41$ & $0.07 \pm 0.32$ & $1.55 \pm 0.39$ & 2 & $1.82 \pm 0.62$ \\
\hline
\end{tabular}

${ }^{a}$ Data are means unless otherwise indicated.

Table 3: Subgroup characteristics defined by their working memory test $z$ scores (DSB $<1$ or $\geq 1$ ) and time since injury ( $<2$ weeks or $2-4$ weeks)

\begin{tabular}{|c|c|c|c|c|c|c|}
\hline & \multicolumn{3}{|c|}{ DSB $<1$} & \multicolumn{3}{|c|}{ DSB $\geq 1$} \\
\hline & MTBI (<2 Weeks) & MTBI (2-4 Weeks) & $\mathrm{HC}$ & MTBI (<2 Weeks) & MTBI (2-4 Weeks) & $\mathrm{HC}$ \\
\hline No. & 4 & 7 & 12 & 4 & 4 & 8 \\
\hline Age & $30 \pm 6$ & $29 \pm 8$ & $31 \pm 7$ & $37 \pm 8$ & $26 \pm 4$ & $37 \pm 14$ \\
\hline DSB & $-0.67 \pm 0.27$ & $-0.24 \pm 0.25$ & $0.08 \pm 0.38$ & $1.42 \pm 0.5$ & $1.58 \pm 0.32$ & $1.79 \pm 0.71$ \\
\hline
\end{tabular}

${ }^{a}$ Data are means unless otherwise indicated.

Right superior longitudinal fasciculus (SLF)

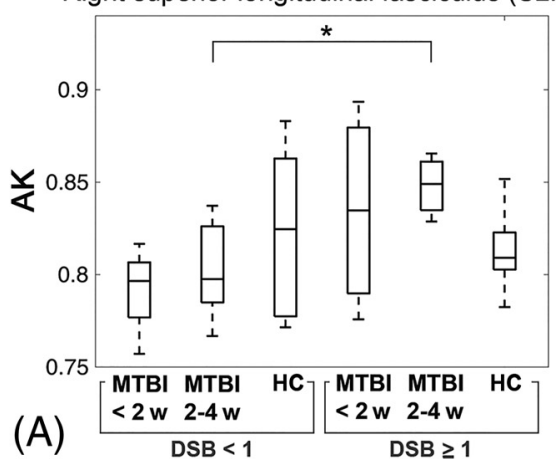

Right posterior corona radiata (CR)

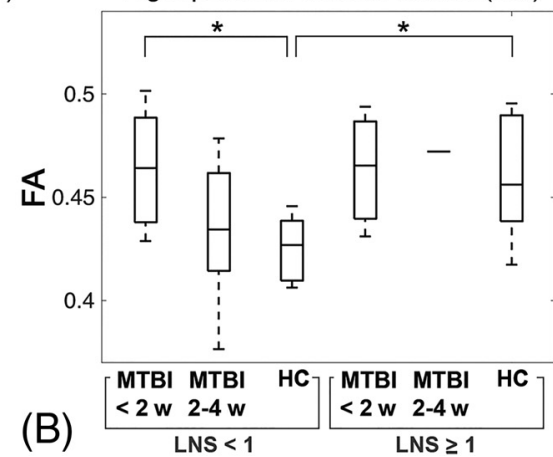

FIG 4. Results of subgroup analysis. A, Boxplots of AK show a significant difference between the MTBI subgroup of 2-4 weeks from injury with DSB $<1$ and the MTBI subgroup of 2-4 weeks from injury with $D S B \geq 1$. $B$, Boxplots of FA show significant differences between the MTBI subgroup of $<2$ weeks of injury with LNS $<1$ and the HC subgroup with LNS $<1$ and between the HC subgroup with LNS $<1$ and the $\mathrm{HC}$ subgroup with LNS $\geq 1$. The asterisk indicates $P<.05 ; \mathrm{w}$, weeks.

poorer performance on DSB. AK is known from animal validation studies to be affected by axonal injury ${ }^{46}$ as well as secondary reactive astrogliosis. ${ }^{47}$

Also differentiating MTBI from HC groups in TBSS analysis was a loss of diffusely present normal associations that we have seen in healthy controls (Figs 2 and 3); in controls, it has been shown that FA and AWF directly correlate with performance on LNS, a complex working memory task. ${ }^{24}$ However, in patients with MTBI, we found a disruption in this normal relationship, presumed to relate to WM injuries known to occur after MTBI, ${ }^{14}$ because AWF is a measure that reflects axonal density and/or myelination.

ROI analyses (Table 1) showed related statistically significant correlations focused in the right SLF in patients with MTBI as well as in the right pCR in healthy controls, compared with the more diffuse TBSS results. TBSS uses maximum values projected onto the WM skeleton, making it more sensitive to maximal deviations in diffusion metrics. Both TBSS and ROI analyses revealed positive correlations in the right SLF and right $\mathrm{pCR}$, suggesting that these regions are strong, potential anatomic landmarks important to working memory performance, and possibly impairment. Along with the SLF, a structure critical to working memory, ${ }^{38-40}$ the corona radiata is also a complex bundle of fibers related to working memory because it consists of several separate pathways connecting the cerebral cortex to

subcortical structures, ${ }^{24,48,49}$ including fiber tracts in the pCR, which connect to the precuneus, a part of the default mode network implicated in working memory performance. $^{50,51}$

With regard to the subgroup analysis, within the same time-since-injury period of 2-4 weeks, there was a significant difference in AK between the MTBI subgroups with higher and lower working memory performance in the right SLF (Fig 4A). Decreased AK, believed to relate to axonal injury ${ }^{46}$ and/or secondary reactive astrogliosis, ${ }^{47}$ may specifically be a more useful indicator in the MTBI subgroup with lower working memory performance. Also, within the lower LNS range, significantly elevated FA was observed in the MTBI subgroup within 2 weeks of injury compared with the corresponding HC subgroup with the same lower LNS range (Fig 4B). Elevated FA was reported frequently within 2 weeks of injury, believed to reflect injury-related cytotoxic edema ${ }^{52}$ or reactive astrogliosis. ${ }^{53}$ We found only a significant difference in FA between the MTBI subgroup within 2 weeks of injury and the HC subgroup, only in the lower LNS range, not in the higher LNS range, suggesting that understanding the relationships between domain-specific symptoms such as working memory deficits and underlying microstructural injuries is important for patient management (eg, pharmacologic intervention to inhibit inflammation and reduce the neurotoxic effects of reactive gliosis).

There are several limitations in the presented study. First, there is a wide age range from 19 to 65 years. Any age effects were minimized using age-corrected WAIS-IV subtest z-scores derived from the published normative sample $(n=2200)$ divided into 13 age bands, spanning ages $16-90 .^{25}$ Moreover, age is included as a covariate in all statistical analyses. Second, subgroups were defined on the basis of a somewhat arbitrary statistical threshold value for LNS and DSB (Fig 4). Further work could study various performance groups. Third, this study includes a relatively small 
number of total subjects and points toward the need for larger studies of working memory dysfunction and brain injury in patients with MTBI. Fourth, this study did not examine the underlying processes that contribute to working memory tasks such as Digit Span and LNS. Further work could focus on component processes such as attention and maintenance of information. Furthermore, as mentioned previously, TBSS uses maximum values projected onto the WM skeleton along an orthogonal line, making it more sensitive to maximal deviations in diffusion metrics but also reducing the need for image smoothing and alleviating any residual misalignment. ${ }^{54}$ In this study, we used both TBSS and ROI approaches to rigorously assess both diffuse and regional WM.

\section{CONCLUSIONS}

There are differences between patients with MTBI within 4 weeks of injury and healthy controls in terms of the relationships between brain microstructure and working memory performance. These findings may relate to known WM injury and changes in functional organization occurring after MTBI. Further study on the effect of time since injury on working memory performance may provide insight into the temporal dynamics of working memory deficits in patients with MTBI.

Disclosures: Sohae Chung-RELATED: Grant: National Institutes of Health, Comments: R01 NS039135-11, R21 NS090349, P41 EB017183.* Els Fieremans-UNRELATED: Board Membership: MicSi, Comments: No money has been paid to me or my institution, but for full disclosure, I am an advisory board member of MicSi, a company that provides advanced image-processing and denoising tools; Royalties: GE Healthcare, Comments: I received $\$ 2125$ in royalties for patent licensing. My institution, NYU School of Medicine, also received royalties; Stock/Stock Options: MicSi, Comments: No money has been paid to me or my institution for stock, but for full disclosure, I own stock (3\%), as well as NYU School of Medicine, in MicSi, a company that provides advanced image-processing and denoising tools. Joseph F. RathRELATED: Grant: National Institutes of Health/National Institute for Neurological Disorders and Stroke, Comments: This article reports on grant-funded research, R01 NS039135.* Charles J. Morton-UNRELATED: Employment: Massachusetts General Hospital, Comments: I worked here from September 2013 to August 2016 as a research coordinator. Dmitry S. Novikov—UNRELATED: Board Membership: Microstructure Imaging, Comments: no money paid; Stock/Stock Options: Microstructure Imaging, Comments: no money paid. Steven R. Flanagan-UNRELATED: Consultancy: McGovern School of Medicine, Icahn School of Medicine at Mount Sinai, Comments: Consultant to McGovern School of Medicine for an external review of their Department of Physical Medicine and Rehabilitation. Education sessions were provided to Icahn School of Medicine at Mount Sinai; Grants/Grants Pending: Brain Injury Association of America, Comments: grant support for the development of guidelines for traumatic brain injury*; Royalties: Springer, Comments: royalties from a textbook; Travel/Accommodations/Meeting Expenses Unrelated to Activities Listed: American Academy of Physical Medicine and Rehabilitation, Comments: Travel and accommodations were provided for face-to-face meetings, which I attend as part of my responsibilities as a member of the Board of Governors of the American Academy of Physical Medicine and Rehabilitation. Yvonne Lui-RELATED: Grant: National Institutes of Health, Comments: R01 NS039135-11, R21 NS090349, P41 EB017183.* *Money paid to the institution.

\section{REFERENCES}

1. Taylor CA, Bell JM, Breiding MJ, et al. Traumatic brain injury-related emergency department visits, hospitalizations, and deaths: United States, 2007 and 2013. MMWR Surveill Summ 2017;66:1-16 CrossRef Medline

2. Centers for Disease Control and Prevention. Report to Congress on Mild Traumatic Brain Injury in the United States: Steps to Prevent a Serious Public Health Problem. Atlanta: Centers for Disease Control and Prevention; 2003

3. Røe C, Sveen U, Alvsåker K, et al. Post-concussion symptoms after mild traumatic brain injury: influence of demographic factors and injury severity in a 1-year cohort study. Disabil Rehabil 2009;31: 1235-43 CrossRef Medline

4. Chen $\mathrm{CJ}, \mathrm{Wu} \mathrm{CH}$, Liao YP, et al. Working memory in patients with mild traumatic brain injury: functional MR imaging analysis. $R a$ diology 2012;264:844-51 CrossRef Medline

5. Ruff R. Two decades of advances in understanding of mild traumatic brain injury. J Head Trauma Rehabil 2005;20:5-18 Medline

6. Mayer AR, Ling J, Mannell MV, et al. A prospective diffusion tensor imaging study in mild traumatic brain injury. Neurology 2010;74: 643-50 CrossRef Medline

7. Niogi SN, Mukherjee P, Ghajar J, et al. Extent of microstructural white matter injury in postconcussive syndrome correlates with impaired cognitive reaction time: a $3 \mathrm{~T}$ diffusion tensor imaging study of mild traumatic brain injury. AJNR Am J Neuroradiol 2008; 29:967-73 CrossRef Medline

8. Rutgers DR, Toulgoat F, Cazejust J, et al. White matter abnormalities in mild traumatic brain injury: a diffusion tensor imaging study. AJNR Am J Neuroradiol 2008;29:514-19 CrossRef Medline

9. Stokum JA, Sours C, Zhuo J, et al. A longitudinal evaluation of diffusion kurtosis imaging in patients with mild traumatic brain injury. Brain Inj 2015;29:47-57 CrossRef Medline

10. Grossman EJ, Jensen JH, Babb JS, et al. Cognitive impairment in mild traumatic brain injury: a longitudinal diffusional kurtosis and perfusion imaging study. AJNR Am J Neuroradiol 2013;34:951-57, S1-3 CrossRef Medline

11. Fieremans E, Jensen JH, Helpern JA. White matter characterization with diffusional kurtosis imaging. Neuroimage 2011;58:177-88 CrossRef Medline

12. Sandry J, Chiou KS, DeLuca J, et al. Individual differences in working memory capacity predicts responsiveness to memory rehabilitation after traumatic brain injury. Arch Phys Med Rehabil 2016;97: 1026-29 e1 CrossRef Medline

13. van der Horn HJ, Liemburg EJ, Scheenen ME, et al. Post-concussive complaints after mild traumatic brain injury associated with altered brain networks during working memory performance. Brain Imaging Behav 2016;10:1243-53 CrossRef Medline

14. McAllister TW, Flashman LA, McDonald BC, et al. Mechanisms of working memory dysfunction after mild and moderate TBI: evidence from functional MRI and neurogenetics. J Neurotrauma 2006; 23:1450-67 CrossRef Medline

15. McDowell S, Whyte J, D'Esposito M. Working memory impairments in traumatic brain injury: evidence from a dual-task paradigm. Neuropsychologia 1997;35:1341-53 CrossRef Medline

16. Hoofien D, Gilboa A, Vakil E, et al. Traumatic brain injury (TBI) 10-20 years later: a comprehensive outcome study of psychiatric symptomatology, cognitive abilities and psychosocial functioning. Brain Inj 2001;15:189-209 CrossRef Medline

17. Kayser AS, Ballard ME, D’Esposito M. Working memory and TBI. In: Alloway TP, ed. Working Memory and Clinical Developmental Disorders: Theories, Debates and Interventions. Abingdon: Routledge. 2018:180-95

18. Baddeley A. Working memory: looking back and looking forward. Nat Rev Neurosci 2003;4:829-39 CrossRef Medline

19. Baddeley A. Working memory: theories, models, and controversies. Annu Rev Psychol 2012;63:1-29 CrossRef Medline

20. Klauer KC, Zhao Z. Double dissociations in visual and spatial shortterm memory. J Exp Psychol Gen 2004;133:355-81 CrossRef Medline

21. Takeuchi H, Taki Y, Sassa Y, et al. Verbal working memory performance correlates with regional white matter structures in the frontoparietal regions. Neuropsychologia 2011;49:3466-73 CrossRef Medline

22. Golestani AM, Miles L, Babb J, et al. Constrained by our connections: white matter's key role in interindividual variability in visual working memory capacity. J Neurosci 2014;34:14913-18 CrossRef Medline

23. Lazar M. Working memory: how important is white matter? Neuroscientist 2017;23:197-210 CrossRef Medline 
24. Chung S, Fieremans E, Kucukboyaci NE, et al. Working memory and brain tissue microstructure: white matter tract integrity based on multi-shell diffusion MRI. Sci Rep 2018;8:3175 CrossRef Medline

25. Wechsler D. Wechsler Adult Intelligence Scale. San Antonio: Pearson; 2008

26. Klonoff PS, Costa LD, Snow WG. Predictors and indicators of quality of life in patients with closed-head injury. J Clin Exp Neuropsychol 1986;8:469-85 CrossRef Medline

27. Chung S, Fieremans E, Wang X, et al. White matter tract integrity: an indicator of axonal pathology after mild traumatic brain injury. J Neurotrauma 2018;35:1015-20 CrossRef Medline

28. Setsompop K, Gagoski BA, Polimeni JR, et al. Blipped-controlled aliasing in parallel imaging for simultaneous multislice echo planar imaging with reduced g-factor penalty. Magn Reson Med 2012;67: 1210-24 CrossRef Medline

29. Veraart J, Fieremans E, Novikov DS. Diffusion MRI noise mapping using random matrix theory. Magn Reson Med 2016;76:1582-93 CrossRef Medline

30. Kellner E, Dhital B, Kiselev VG, et al. Gibbs-ringing artifact removal based on local subvoxel-shifts. Magn Reson Med 2016;76:1574-81 CrossRef Medline

31. Collier Q, Veraart J, Jeurissen B, et al. Iterative reweighted linear least squares for accurate, fast, and robust estimation of diffusion magnetic resonance parameters. Magn Reson Med 2015;73:2174-84 CrossRef Medline

32. Smith SM, Jenkinson M, Johansen-Berg H, et al. Tract-based spatial statistics: voxelwise analysis of multi-subject diffusion data. Neuroimage 2006;31:1487-505 CrossRef Medline

33. Jensen JH, McKinnon ET, Glenn GR, et al. Evaluating kurtosisbased diffusion MRI tissue models for white matter with fiber ball imaging. NMR Biomed 2017;30 CrossRef Medline

34. Mori S, Oishi K, Jiang H, et al. Stereotaxic white matter atlas based on diffusion tensor imaging in an ICBM template. Neuroimage 2008;40:570-82 CrossRef Medline

35. Fisher RA. Statistical Methods for Research Workers. Edinburgh: Oliver and Boyd; 1948

36. Mesulam MM. From sensation to cognition. Brain 1998;121(Pt 6): 1013-52 CrossRef Medline

37. Petrides M, Pandya DN. Comparative cytoarchitectonic analysis of the human and the macaque ventrolateral prefrontal cortex and corticocortical connection patterns in the monkey. Eur J Neurosci 2002;16:291-310 CrossRef Medline

38. Cohen JD, Perlstein WM, Braver TS, et al. Temporal dynamics of brain activation during a working memory task. Nature 1997;386: 604-08 CrossRef Medline

39. Prabhakaran V, Narayanan $\mathrm{K}$, Zhao Z, et al. Integration of diverse information in working memory within the frontal lobe. Nat Neurosci 2000;3:85-90 CrossRef Medline

40. Todd JJ, Marois R. Capacity limit of visual short-term memory in human posterior parietal cortex. Nature 2004;428:751-54 CrossRef Medline

41. Frye RE, Hasan K, Malmberg B, et al. Superior longitudinal fasciculus and cognitive dysfunction in adolescents born preterm and at term. Dev Med Child Neurol 2010;52:760-66 CrossRef Medline

42. Hoeft F, Barnea-Goraly N, Haas BW, et al. More is not always better: increased fractional anisotropy of superior longitudinal fasciculus associated with poor visuospatial abilities in Williams syndrome. J Neurosci 2007;27:11960-65 CrossRef Medline

43. Chechlacz M, Gillebert CR, Vangkilde SA, et al. Structural variability within frontoparietal networks and individual differences in attentional functions: an approach using the theory of visual attention. J Neurosci 2015;35:10647-58 CrossRef Medline

44. Palacios EM, Fernandez-Espejo D, Junque C, et al. Diffusion tensor imaging differences relate to memory deficits in diffuse traumatic brain injury. BMC Neurol 2011;11:24 CrossRef Medline

45. Jensen JH, Helpern JA. MRI quantification of non-Gaussian water diffusion by kurtosis analysis. NMR Biomed 2010;23:698-710 CrossRef Medline

46. Andersson $G$, Orädd $G$, Sultan F, et al. In vivo diffusion tensor imaging, diffusion kurtosis imaging, and tractography of a sciatic nerve injury model in rat at 9.4T. Sci Rep 2018;8:12911 CrossRef Medline

47. Zhuo J, Xu S, Proctor JL, et al. Diffusion kurtosis as an in vivo imaging marker for reactive astrogliosis in traumatic brain injury. Neuroimage 2012;59:467-77 CrossRef Medline

48. Tang CY, Eaves EL, Ng JC, et al. Brain networks for working memory and factors of intelligence assessed in males and females with $\mathrm{fMRI}$ and DTI. Intelligence 2010;38:293-303 CrossRef

49. Long YC, Ouyang X, Liu ZN, et al. Associations among suicidal ideation, white matter integrity and cognitive deficit in first-episode schizophrenia. Front Psychiatry 2018;9:391 CrossRef Medline

50. Esposito F, Aragri A, Latorre V, et al. Does the default-mode functional connectivity of the brain correlate with working-memory performances? Arch Ital Biol 2009;147:11-20 Medline

51. Bluhm RL, Clark CR, McFarlane AC, et al. Default network connectivity during a working memory task. Hum Brain Mapp 2011;32: 1029-35 CrossRef Medline

52. Shenton ME, Hamoda HM, Schneiderman JS, et al. A review of magnetic resonance imaging and diffusion tensor imaging findings in mild traumatic brain injury. Brain Imaging Behav 2012;6:137-92 CrossRef Medline

53. Budde MD, Janes L, Gold E, et al. The contribution of gliosis to diffusion tensor anisotropy and tractography following traumatic brain injury: validation in the rat using Fourier analysis of stained tissue sections. Brain 2011;134:2248-60 CrossRef Medline

54. Bach M, Laun FB, Leemans A, et al. Methodological considerations on tract-based spatial statistics (TBSS). Neuroimage 2014;100: 358-69 CrossRef Medline 